\title{
PERTURBATIONS BY NILPOTENT OPERATORS ON HILBERT SPACE
}

\author{
ARLEN BROWN, CARL PEARCY AND NORBERTO SALINAS ${ }^{1}$
}

ABstract. If $T$ is any noncompact, bounded, linear operator on a separable Hilbert space $H$, then there exists a nilpotent (bounded, linear) operator $N$ on $H$ such that $N+T$ is invertible.

1. Introduction. If $A$ is a nonzero operator on a finite dimensional, complex Hilbert space $\mathscr{K}$, then it is an exercise in linear algebra (cf. [2]) to find a nilpotent operator $N$ on $\mathscr{K}$ such that $A+N$ is invertible. The object of this note is to generalize this result to operators acting on a Hilbert space of dimension $\aleph_{0}$. Thus, throughout the remainder of this paper, $\mathscr{H}$ will denote a separable, infinite dimensional, complex Hilbert space, and $\mathscr{L}(\mathscr{H})$ will denote the algebra of all (bounded, linear) operators on $\mathscr{H}$. As it turns out, the appropriate generalization of the above-mentioned fact to operators on $\mathscr{H}$ provides a novel characterization of the ideal of compact operators in $\mathscr{L}(\mathscr{H})$. Our main result is the following theorem.

THEOREM 1.1. An operator $A$ on $\mathscr{H}$ fails to be compact if and only if there exists a nilpotent operator $N$ on $\mathscr{H}$ such that $A+N$ is invertible.

This theorem may be regarded as a generalization of the finite-dimensional result mentioned above because the ideal of compact operators is the maximal proper ideal in $\mathscr{L}(\mathscr{H})$ and $(0)$ is the maximal proper ideal in the ring of operators on a finite dimensional space. Thus, each result says that every operator that is not in the maximal proper ideal has a nilpotent perturbation which is invertible.

In $\S 4$ the proof of Theorem 1.1 will be given. At this point, we remark that an immediate consequence of this theorem is the following corollary, which is due to Dyer, Porcelli, and Rosenfeld [2, Corollary B].

COROLlary 1.2. An operator $A$ on $\mathscr{H}$ fails to be compact if and only if there exists $B$ in $\mathscr{L}(\mathscr{H})$ such that the spectrum of $A+B$ is disjoint from the spectrum of $B$.

Received by the editors June 2, 1972 and, in revised form, December 6, 1972.

AMS (MOS) subject classifications (1970). Primary 47A55.

${ }^{1}$ This research was done while the third author was a temporary member of the Institute for Advanced Study; it was supported in part by the National Science Foundation.

(c) American Mathematical Society 1973 
The proof of the nonobvious half of Theorem 1.1 splits naturally into two cases, and we shall consider them separately. We denote by $(J)$ the ideal of all compact operators in $\mathscr{L}(\mathscr{H})$, and by $(S)$ the set of all operators of the form $\lambda+T$ where $T \in(J)$ and $\lambda$ is a nonzero scalar. Also, $(F)$ will denote the complement in $\mathscr{L}(\mathscr{H})$ of $(J) \cup(S)$. In $\S 2$ we give the proof for operators in $(F)$, and in $\S 3$ we treat operators in $(S)$. In each case, slightly more is proved than is necessary to prove Theorem 1.1.

2. Nilpotent perturbations of operators in $(F)$. In what follows, $\Lambda(A)$ will denote the spectrum of an operator $A$.

THEOREM 2.1. Let $A$ be an operator belonging to $(F)$. Then there exists a nilpotent operator $N$ on $\mathscr{H}$ such that $\Lambda(A+N)$ lies outside any prescribed disk in the plane.

Proof. From [1, Theorem 2] it follows that $A$ is similar to an operator $B$ acting on $\mathscr{H} \oplus \mathscr{H} \oplus \mathscr{H}$ whose matrix has the form

$$
B=\left(\begin{array}{ccc}
0 & A_{12} & A_{13} \\
0 & A_{22} & A_{23} \\
1_{\mathscr{H}} & A_{32} & A_{33}
\end{array}\right),
$$

where $A_{i j} \in \mathscr{L}(\mathscr{H}), i=1,2,3, j=2,3$. Since the spectrum of an operator is invariant under similarity, it suffices to prove the theorem for the operator $B$. Let $\rho$ be any positive number, and let $\mathscr{D}$ be the disk $\mathscr{D}=$ $\{\lambda:|\lambda| \leqq \rho\}$ in the complex plane. For each $\alpha>0$ we denote by $N_{\alpha}$ the nilpotent operator on $\mathscr{H} \oplus \mathscr{H} \oplus \mathscr{H}$ given matricially by

$$
N_{\alpha}=\left(\begin{array}{lll}
0 & \alpha & 0 \\
0 & 0 & \alpha \\
0 & 0 & 0
\end{array}\right) \text {, }
$$

where, of course, $\alpha$ denotes the scalar operator $\alpha 1_{\mathscr{H}}$. The proof of the theorem will be completed by showing that there exists a positive number $\beta$ large enough so that $\Lambda\left(B+N_{\beta}\right) \cap \mathscr{D}=\varnothing$. To this end, for each $\alpha>0$, let $S_{\alpha}$ denote the invertible operator matrix

$$
S_{\alpha}=\left(\begin{array}{ccc}
\alpha^{2 / 3} & 0 & 0 \\
0 & \alpha^{1 / 3} & 0 \\
0 & 0 & 1
\end{array}\right)
$$

A matricial calculation shows that $\left(S_{\alpha}\right)^{-1}\left(B+N_{\alpha}\right) S_{\alpha}$ is the matrix

$$
\alpha^{2 / 3}\left[\left(\begin{array}{lll}
0 & 1 & 0 \\
0 & 0 & 1 \\
1 & 0 & 0
\end{array}\right)+\left(\begin{array}{ccc}
0 & A_{12} / \alpha & A_{13} / \alpha^{4 / 3} \\
0 & A_{22} / \alpha^{2 / 3} & A_{23} / \alpha \\
0 & A_{32} / \alpha^{1 / 3} & A_{33} / \alpha^{2 / 3}
\end{array}\right)\right] .
$$


Since the spectrum of the first matrix in (1) clearly lies on the unit circle, and since the second matrix tends to zero in the uniform topology as $\alpha$ goes to infinity, it follows from the upper-semicontinuity of the spectrum that for $\alpha$ large enough, the spectrum of the sum of the two matrices in (1) lies in an arbitrarily thin annulus containing the unit circle in its interior. Thus (by virtue of the factor $\alpha^{2 / 3}$ in (1)) $\beta$ may be chosen sufficiently large so that $\Lambda\left[\left(S_{\beta}\right)^{-1}\left(B+N_{\beta}\right) S_{\beta}\right] \cap \mathscr{D}=\varnothing$, and the theorem follows.

3. Nilpotent perturbations of Fredholm operators. We recall that $A$ is a Fredholm operator of index zero if and only if the null space of $A$ and the null space of $A^{*}$ are finite dimensional subspaces of the same dimension and the range of $A$ is closed. It is well known that if $A$ is a Fredholm operator of index zero on $\mathscr{H}$, then there exists a finite rank operator $F \in \mathscr{L}(\mathscr{H})$ such that $A+F$ is invertible. The following theorem shows that the finite rank operator $F$ mentioned in the above statement can be taken to be nilpotent whenever zero is an isolated point of the spectrum of $A$.

THEOREM 3.1. If $A$ is a Fredholm operator of index zero in $\mathscr{L}(\mathscr{H})$ and zero is an isolated point of $\Lambda(A)$, then there exists a nilpotent, finite rank operator $N$ on $\mathscr{H}$ such that $A+N$ is invertible.

Proof. Let $E_{0}$ and $E_{1}$ be the spectral idempotents for $A$ associated with the sets $\Lambda(A) \backslash\{0\}$ and $\{0\}$, respectively, and for $j=0,1$, let $\mathscr{E}_{j}$ denote the range of $E_{j}$. If we write $A_{j}=A \mid \mathscr{E}_{j}$, then $\Lambda\left(A_{0}\right)=\Lambda(A) \backslash\{0\}$ and $\Lambda\left(A_{1}\right)=$ $\{0\}$. Since $A$ is a Fredholm operator of index zero, it follows that $\mathscr{E}_{1}$ is finite dimensional [3, Lemma 4.1], and hence that $A_{1}$ is a nilpotent operator. It is easy to see that for $j=0,1$, there exist Hilbert spaces $\mathscr{H}_{j}$ and invertible operators $Z_{j}: \mathscr{H}_{j} \rightarrow \mathscr{E}_{j}$ such that the bounded operator $Z: \mathscr{H}_{0} \oplus \mathscr{H}_{1} \rightarrow \mathscr{H}$ defined by $Z \mid \mathscr{H}_{j}=Z_{j}(j=0,1)$ is invertible and satisfies $Z^{-1} A Z=B_{0} \oplus B_{1}$, where $B_{j}=Z_{j}^{-1} A_{j} Z_{j}(j=0,1)$. In view of the above remark, in order to complete the proof of the theorem, it suffices to find a nilpotent, finite rank operator

$$
\left(\begin{array}{cc}
L_{0} & M_{0} \\
M_{1} & L_{1}
\end{array}\right)
$$

acting on $\mathscr{H}_{0} \oplus \mathscr{H}_{1}$ such that

$$
\left(\begin{array}{cc}
B_{0} & 0 \\
0 & B_{1}
\end{array}\right)+\left(\begin{array}{cc}
L_{0} & M_{0} \\
M_{1} & L_{1}
\end{array}\right)
$$

is invertible. Let $V: \mathscr{H}_{1} \rightarrow \mathscr{H}_{0}$ be any isometry and define

$$
\left(\begin{array}{cc}
L_{0} & M_{0} \\
M_{1} & L_{1}
\end{array}\right)=\left(\begin{array}{cc}
-V V^{*} & -V \\
V^{*} & 1-B_{1}
\end{array}\right)
$$


Since $\mathscr{H}_{1}$ is finite dimensional, it is clear that the matrix in (2) represents a finite rank operator. Furthermore, since

$$
\left(\begin{array}{cc}
-V V^{*} & -V \\
V^{*} & 1-B_{1}
\end{array}\right)^{2}=\left(\begin{array}{ll}
V & 0 \\
0 & 1
\end{array}\right)\left(\begin{array}{cc}
B_{1} & 0 \\
0 & B_{1}
\end{array}\right)\left(\begin{array}{cc}
0 & 1 \\
-1 & B_{1}-2
\end{array}\right)\left(\begin{array}{cc}
V^{*} & 0 \\
0 & 1
\end{array}\right),
$$

and since $B_{1}$ is a nilpotent operator, a brief calculation shows that the matrix in (2) is nilpotent. Finally, from the following elementary lemma, we conclude that the matrix defined in (2) satisfies all the required conditions.

Lemma 3.2. Let $C_{j}$ be an element of $\mathscr{L}\left(\mathscr{H}_{j}\right), j=0,1$, such that $C_{1}$ is invertible, and for $j=0,1$, let $D_{j}: \mathscr{H}_{1-j} \rightarrow \mathscr{H}_{j}$ be a bounded linear transformation. Then the operator matrix

$$
\left(\begin{array}{ll}
C_{0} & D_{0} \\
D_{1} & C_{1}
\end{array}\right)
$$

is invertible if and only if $C_{0}-D_{0} C_{1}^{-1} D_{1}$ is an invertible operator on $\mathscr{H}_{0}$.

Proof. Since the operator matrices

$$
\left(\begin{array}{cc}
1_{\mathscr{H}_{0}} & -D_{0} C_{1}^{-1} \\
0 & 1_{\mathscr{H}_{1}}
\end{array}\right) \quad \text { and } \quad\left(\begin{array}{cc}
1_{\mathscr{H}_{0}} & 0 \\
0 & C_{1}^{-1}
\end{array}\right)
$$

are invertible, it follows that

is invertible if and only if

$$
\left(\begin{array}{ll}
C_{0} & D_{0} \\
D_{1} & C_{1}
\end{array}\right)
$$

$$
\begin{array}{r}
\left(\begin{array}{cc}
1_{\mathscr{H}_{0}} & -D_{0} C_{1}^{-1} \\
0 & 1_{\mathscr{H}_{1}}
\end{array}\right)\left(\begin{array}{cc}
C_{0} & D_{0} \\
D_{1} & C_{1}
\end{array}\right)\left(\begin{array}{cc}
1_{\mathscr{H}_{0}} & 0 \\
0 & C_{1}^{-1}
\end{array}\right) \\
=\left(\begin{array}{ccc}
C_{0} & -D_{0} C_{1}^{-1} D_{1} & 0 \\
D_{1} & 1_{\mathscr{H}_{1}}
\end{array}\right)
\end{array}
$$

enjoys the same property. On the other hand, an easy matricial computation shows that the matrix on the right-hand side of (3) is invertible if and only if the same condition holds for the operator $C_{0}-D_{0} C_{1}^{-1} D_{1}$.

4. Proof of the main result. Given an operator $A$ on $\mathscr{H}$ we denote by $\Lambda_{\text {ess }}(A)$ the essential spectrum of $A$, i.e., the spectrum of the image of $A$ under the canonical map from $\mathscr{L}(\mathscr{H})$ onto the quotient algebra $\mathscr{L}(\mathscr{H}) /(J)$. 
Proof of Theorem 1.1. If $A$ is a compact operator and $N$ is a nilpotent operator on $\mathscr{H}$, then $\Lambda(A+N) \supset \Lambda_{\text {ess }}(A+N)=\Lambda_{\text {ess }}(N)=\{0\}$ and hence $A+N$ is not invertible. Conversely, suppose that $A \in(F) \cup(S)$. Suppose further, as we may without loss of generality, that $A$ is not invertible (if $A$ is invertible, set $N=0$ ). If $A \in(S)$, then $A$ is a Fredholm operator of index zero such that zero is an isolated point of $\Lambda(A)$, and the desired operator $N$ is provided by Theorem 3.1. Since the case in which $A \in(F)$ is covered by Theorem 2.1, the proof of Theorem 1.1 is complete.

\section{REFERENCES}

1. A. Brown and C. Pearcy, Structure of commutators of operators, Ann. of Math. (2) 82 (1965), 112-127. MR 31 \#2612.

2. J. Dyer, P. Porcelli and M. Rosenfeld, Spectral characterization of two sided ideals in $B(H)$, Israel J. Math. 10 (1971), 26-31.

3. N. Salinas, Operators with essentially disconnected spectrum, Acta. Sci. Math. (Szeged) 33 (1972), 193-205.

Department of Mathematics, Indiana University, Bloomington, Indiana 47401

Department of Mathematics, University of Michigan, Ann Arbor, Michigan 48104

Department of Mathematics, University of Kansas, LaWrence, Kansas 66044 